Quim. Nova, Vol. 31, No. 5, 1094-1098, 2008

\title{
DETERMINAÇÃo DE PALÁDIO EM AMOSTRAS BIOLÓGICAS APLICANDO TÉCNICAS ANALÍTICAS NUCLEARES
}

\author{
Cássio Q. Cavalcante*, Ivone M. Sato, Vera L. R. Salvador e Mitiko Saiki \\ Departamento de Análise por Ativação Neutrônica, Instituto de Pesquisas Energéticas e Nucleares, Av. Prof. Lineu Prestes, \\ 2242, 05508-000 São Paulo - SP, Brasil
}

Recebido em 9/5/07; aceito em 24/11/07; publicado na web em 8/7/08

\begin{abstract}
DETERMINATION OF PALLADIUM IN BIOLOGICAL SAMPLES APPLYING NUCLEAR ANALYTICAL TECHNIQUES. This study presents Pd determinations in bovine tissue samples containing palladium prepared in the laboratory, and CCQM-P63 automotive catalyst materials of the Proficiency Test, using instrumental thermal and epithermal neutron activation analysis and energy dispersive X-ray fluorescence techniques. Solvent extraction and solid phase extraction procedures were also applied to separate Pd from interfering elements before the irradiation in the nuclear reactor. The results obtained by different techniques were compared against each other to examine sensitivity, precision and accuracy.
\end{abstract}

Keywords: nuclear analytical techniques; palladium; biological samples.

\section{INTRODUÇÃO}

O paládio é um elemento presente em baixas concentrações na natureza. Entretanto, com o seu crescente uso em catalisadores automotivos para conversão do monóxido de carbono, é hoje considerado como um novo contaminante do meio ambiente. Conseqüentemente, a determinação de Pd é requerida em amostras biológicas para avaliar os efeitos deste elemento à saúde humana.

O Pd também tem sido estudado para uso na preparação de novas drogas antitumorais devido à manifestação menor de efeitos colaterais. ${ }^{1,2}$ Desta forma para avaliar a biodistribuição, eficiência e os efeitos colaterais destas drogas contendo Pd torna-se de grande importância o estudo sobre métodos analíticos para a determinação de Pd em amostras biológicas.

Entre os diversos métodos existentes para a determinação de Pd os mais utilizados são a análise por ativação com nêutrons (NAA), ${ }^{3-8}$ a espectroscopia de massa com fonte de plasma acoplado indutivamente (ICP-MS), ${ }^{9-16}$ de absorção atômica (AAS), ${ }^{12,17-20}$ de emissão óptica (OES), ${ }^{21}$ de absorção atômica em forno de grafite $(\text { GF-AAS })^{22-24}$ e de fluorescência de raios X (XRF). ${ }^{25,26}$ As aplicações destas técnicas geralmente estão restritas à análise de amostras com nenhuma ou com baixa interferência na análise, de modo que comumente são utilizadas a pré-separação do $\mathrm{Pd}$ dos elementos interferentes e também a pré-concentração.

Para a determinação do Pd pela NAA o radioisótopo mais adequado para sua medição é o ${ }^{109} \mathrm{Pd}$ com meia vida de 13,7 h e energia de raios gama de $88,03 \mathrm{keV}$ devido às características nucleares do isótopo alvo serem mais favoráveis para ativação. Embora a NAA esteja bem estabelecida para determinação de diversos elementos em concentrações traço e ultratraço em diferentes tipos de matrizes, a determinação de Pd em amostras biológicas por este método apresenta o problema de interferências. A alta atividade do ${ }^{24} \mathrm{Na}$, formado pela ativação com nêutrons do sódio presente em alto teor na amostra, impede a medição do paládio devido à saturação do detector e, também, o efeito Compton do ${ }^{24} \mathrm{Na}$ e da radiação de freiamento do ${ }^{32} \mathrm{P}$ mascaram as atividades menos intensas do ${ }^{109} \mathrm{Pd}$. Além disso, como o pico de ${ }^{109} \mathrm{Pd}$ a ser medido é de baixa

*e-mail: cavalcante.cassio@gmail.com energia $(88,03 \mathrm{keV})$ ocorre a interferência de picos de raios $\mathrm{X}$.

Entre as várias técnicas existentes para a separação ou pré-concentração de $\mathrm{Pd}$, as mais utilizadas têm sido a extração com solvente, ${ }^{5-7,16}$ a extração em fase sólida, ${ }^{3,12,17,27}$ a resina de troca iônica ${ }^{8,20,28}$ e a pré-concentração do Pd em sulfeto de níquel, obtido por meio de ensaio de fogo, seguida de co-precipitação em telúrio metálico. ${ }^{14,15,29}$

São apresentados neste trabalho os resultados da determinação de Pd em amostras biológicas e material catalisador automotivo obtidos pelos métodos de ativação instrumental com nêutrons térmicos e epitérmicos separadamente e pela técnica de fluorescência de raios X por dispersão de energia (EDXRF). A análise por ativação foi realizada com e sem separação prévia do Pd dos elementos interferentes. Os métodos de pré-separação utilizados foram a extração com solventes ${ }^{6}$ e extração em fase sólida. ${ }^{3}$

\section{PARTE EXPERIMENTAL}

\section{Preparação de amostras de tecido bovino contendo paládio}

Devido a não disponibilidade para aquisição de materiais biológicos de referência com valores certificados para o $\mathrm{Pd}^{30}$ para uso no estudo da determinação deste elemento, foram preparadas amostras biológicas contendo Pd adicionado. Foram adquiridas amostras de tecido muscular e de fígado bovino para esta preparação. A partir do tecido bovino livre de tecido adiposo foram preparadas sub-amostras com e sem Pd adicionado. Na preparação da amostra biológica com Pd adicionado, para $120 \mathrm{~g}$ de tecido bovino foram adicionados $70 \mathrm{~mL}$ de solução de paládio com a concentração de $11,4 \mathrm{mg} \mathrm{L}^{-1}$ e efetuou-se a sua homogeneização usando liquidificador doméstico com lâmina de titânio. A seguir, as porções de tecido muscular sem e com Pd foram congeladas e posteriormente secas utilizando o liofilizador da marca Micro Modulyo. Neste processo de secagem por liofilização foram verificadas para as porções sem e com Pd perdas de peso de 87,3 e 75,1\%, respectivamente. Após a liofilização as amostras secas foram trituradas e homogeneizadas em recipiente de teflon.

No caso da amostra de fígado o procedimento da preparação das porções sem e com Pd adicionado foi o mesmo adotado para o 
caso do músculo bovino. Para a amostra sem Pd em $185 \mathrm{~g}$ de fígado cortado em pedaços foram adicionados $50 \mathrm{~mL}$ de água Milli-Q. $\mathrm{Na}$ preparação da amostra com Pd adicionado, a $150 \mathrm{~g}$ de fígado cortado em pedaços foram adicionados $40 \mathrm{~mL}$ de solução de paládio com a concentração de $9,8 \mathrm{mg} \mathrm{L}^{-1}$. Na liofilização obteve-se perdas de peso de 85,4 e $86,3 \%$, para as amostras de fígado bovino sem e com Pd adicionado, respectivamente.

Neste trabalho foi analisado também o material catalisador automotivo CCQM-P63 do teste de proficiência do Comité Consultatif pour la Quantité de Matière (CCQM).

\section{Preparação do padrão sintético de paládio}

Para a preparação do padrão sintético de paládio para uso na análise por ativação com nêutrons utilizou-se solução de paládio certificado com a concentração de $1002,5 \pm 3 \mathrm{mg} \mathrm{L}^{-1}$ adquirida da SpexCertiPrep, EUA. Esta solução foi diluída com água purificada Milli-Q obtendo-se uma solução de Pd com concentração de 100,25 $\mu \mathrm{g} \mathrm{mL}{ }^{-1}$. Pipetou-se $50 \mu \mathrm{L}$ desta solução diluída em tiras de papel de filtro Whatmam $n^{\circ} 40$, as quais foram colocadas em um dessecador para a secagem da solução pipetada por $24 \mathrm{~h}$. Cada uma das tiras de papel de filtro dobradas com o auxílio de pinças foi colocada em invólucro de polietileno para irradiação no reator juntamente com a amostra.

\section{Métodos analíticos}

Análise instrumental por ativação com nêutrons térmicos (NAA) e epitérmicos (ENAA)

$\mathrm{Na}$ análise instrumental por NAA e ENAA foram utilizadas cerca de 100 a $200 \mathrm{mg}$ de cada amostra, pesadas em invólucros de polietileno usando balança analítica da marca Mettler modelo $\mathrm{H}$ 16. O padrão de $\mathrm{Pd}$ e as amostras colocados em invólucros de polietileno foram envoltos individualmente em folhas de alumínio e acondicionados em um dispositivo de alumínio para irradiação chamado de "coelho". Nas irradiações epitérmicas este conjunto de padrão e amostras, antes de ser acondicionado no "coelho" de alumínio, foi colocado em cápsula de cádmio com $2 \mathrm{~mm}$ de espessura. As irradiações foram realizadas no reator nuclear de pesquisa IEA-R1 por um período de $4 \mathrm{~h}$ nas irradiações térmicas e $16 \mathrm{~h}$ nas irradiações epitérmicas, sob fluxo de nêutrons térmicos da ordem de $4 \times 10^{12} \mathrm{n} \mathrm{cm}^{-2} \mathrm{~s}^{-1}$. Após tempos de decaimento de aproximadamente $60 \mathrm{~h}$, foi realizada a abertura do dispositivo de irradiação. O padrão de Pd e as amostras irradiadas foram montados individualmente em suportes de aço inoxidável, fixando-os com fita colante para as medições das atividades gama induzidas. Estas medições das radiações gama das amostras e do padrão foram feitas usando espectrômetro de raios gama ligado a um detector de Ge hiperpuro e a sistema eletrônico associado. A resolução do sistema utilizado foi de $0,98 \mathrm{keV}$ para o fotopico de $122,1 \mathrm{keV}$ do ${ }^{57} \mathrm{Co}$ e de $1,78 \mathrm{keV}$ para o fotopico de $1332,5 \mathrm{keV}$ do ${ }^{60} \mathrm{Co}$. A geometria para medição no detector foi escolhida de modo que o tempo morto seja inferior a $15 \%$, para evitar o efeito da saturação do detector. Os tempos de contagem variaram de 1800 a $3600 \mathrm{~s}$ para os padrões e de 1800 a $12000 \mathrm{~s}$ para as amostras, dependendo da atividade da amostra. Por meio do programa S100 da Canberra, foram obtidos os dados espectrais e usando o programa Versão 2 foram processados os espectros de raios gama, obtendo-se as energias dos raios gama dos radioisótopos formados e os valores das taxas de contagens (área sob os picos). Foi feita a medição da radioatividade gama do radioisótopo ${ }^{109} \mathrm{Pd}$, que apresenta tempo de meia vida de $13,7 \mathrm{~h}$ e emite raios-gama energia de $88,0 \mathrm{keV}$. A concentração de paládio foi determinada pelo método comparativo.
Análise por ativação com nêutrons epitérmicos (ENAA) após a pré-separação do paládio

\section{Digestão da amostra}

Cerca de $300 \mathrm{mg}$ da amostra pesados diretamente em frasco de teflon da Savilex foram dissolvidos com $4 \mathrm{~mL}$ de $\mathrm{HNO}_{3}$ concentrado e mantidos por 5,5 h em contato. Em seguida, foi adicionado 1 $\mathrm{mL}$ de $\mathrm{H}_{2} \mathrm{O}_{2}$ e deixado reagir durante toda a noite. Após o pernoite, esta amostra foi digerida durante $3 \mathrm{~h}$ em um bloco digestor da Tecnal a temperatura de $90{ }^{\circ} \mathrm{C}$. Após este tempo, o frasco com a amostra dissolvida foi deixado a temperatura ambiente até o seu completo resfriamento e posterior separação do Pd dos elementos interferentes.

\section{Pré-separação do paládio pela técnica de extração com solventes}

O procedimento para a pré-separação do Pd pela técnica de extração com solventes, foi baseado no trabalho de Byrne. ${ }^{6}$ Transferiu-se a solução obtida na digestão em um funil de separação onde foram adicionados $1 \mathrm{~mL}$ de ácido etileno diamino tetraacético (EDTA) $0,1 \mathrm{M}$ e 0,5 mL de solução de dimetilglioxima (DMG) $1 \%$ dissolvido em etanol. Agitou-se a mistura do funil e deixou-se por no mínimo $15 \mathrm{~min}$ em repouso. A seguir, a esta mistura foram adicionados $5 \mathrm{~mL}$ de clorofórmio e a extração foi feita por agitação manual e a separação das fases por decantação. Recolheu-se a fase orgânica e repetiu-se a operação de extração com a adição de mais 2,5 mL de clorofórmio. Combinaram-se as fases orgânicas recolhidas nas duas operações e foi feita a lavagem com duas porções de $5 \mathrm{~mL}$ de ácido clorídrico 0,2 M. Recolheu-se a fase orgânica em balão volumétrico de $10 \mathrm{~mL}$ e completou-se o volume com clorofórmio. Alíquota de $250 \mu \mathrm{L}$ da solução orgânica resultante foi pipetada em cápsula de polietileno de alta pureza da Vrije Universiteit Amsterdam, The Netherlands e submetida à secagem sob uma lâmpada de raios infravermelho. Estas cápsulas foram seladas e irradiadas com nêutrons epitérmicos, juntamente com um padrão sintético de Pd também pipetado e seco em uma cápsula. As condições de irradiação no reator nuclear foram as mesmas adotadas na análise por ativação instrumental com nêutrons epitérmicos.

\section{Pré-separação pela técnica de extração em fase sólida}

Na pré-separação do Pd pela técnica de extração em fase sólida, o procedimento foi baseado no trabalho de Schwarzer et al. ${ }^{3} \mathrm{~A}$ preparação do cartucho de extração de fase sólida, da marca Phenomenex modelo SPE-Strata C18-E, foi feita por meio da adsorção de $10 \mathrm{mg}$ de complexante N,N-dietil- N'-benzoil tiouréia (DEBT) dissolvidos na concentração de $1 \%$ de DEBT em mistura de água e metanol na proporção 0,9:1 (v/v). Os cartuchos com o complexante adsorvido foram lavados usando-se $5 \mathrm{~mL}$ de água purificada Milli-Q antes da percolação da solução da amostra. A retenção de Pd no cartucho foi realizada pela percolação da solução obtida na dissolução da amostra e, em seguida, de $5 \mathrm{~mL}$ de ácido nítrico 6,5\% para eliminar os interferentes. O complexo de Pd formado na coluna foi eluído com $10 \mathrm{~mL}$ de etanol. Para análise, alíquotas de $250 \mu \mathrm{L}$ da solução eluída foram pipetadas e secas em cápsulas de polietileno e irradiados com nêutrons epitérmicos juntamente com o padrão de Pd.

Análise pela técnica de fluorescência de raios $X$ por dispersão de energia (EDXRF)

A determinação de Pd por EDXRF foi realizada utilizando o espectrômetro da Shimaduz Co, modelo Rayny e método de adição. Foram adicionadas quantidades conhecidas de $\mathrm{Pd}$, a partir de 
solução padrão certificada da Aldrich $\left(998 \pm 48 \mathrm{mg} \mathrm{kg}^{-1}\right)$, no material certificado de fígado bovino (SRM $1577 \mathrm{~b}$ da NIST). As frações desse material foram secas no dessecador por $48 \mathrm{~h} \mathrm{e}$ homogeneizadas em almofariz de ágata por $40 \mathrm{~min}$. A curva de calibração foi obtida inter-relacionando as concentrações adicionadas com as relações das intensidades fluorescentes $\mathrm{PdKa} / \mathrm{BG}$, para compensar o efeito de espalhamento de raios $\mathrm{X}$ na análise das amostras em forma de pó solto.

\section{RESULTADOS E DISCUSSÃO}

As médias das determinações de paládio das amostras de tecido muscular e fígado bovino por meio de diferentes técnicas analíticas nucleares são apresentadas na Tabela 1. Estes resultados apresentam, em geral, uma boa reprodutibilidade com desvios padrão relativos variando de 1,2 a $14,0 \%$. Os resultados menos precisos foram obtidos pelo método instrumental de análise por ativação com nêutrons térmicos. Os métodos envolvendo a pré-separação química permitiram a obtenção de resultados altamente satisfatórios com relação à precisão.

Tabela 1. Concentrações de Pd obtidas na análise de amostras biológicas de tecido muscular e fígado bovino, por diferentes técnicas

\begin{tabular}{lcccc}
\hline Amostra & $\begin{array}{c}\text { Técnica de } \\
\text { pré-concentração/ } \\
\text { separação }\end{array}$ & $\begin{array}{c}\text { Método } \\
\text { de análise }\end{array}$ & $\begin{array}{c}\mathrm{Pd} \\
\mathrm{X} \pm \mathrm{DP}^{\mathrm{b}}(\mathrm{n})^{\mathrm{c}} \\
\left.(\mu \mathrm{g} \mathrm{g})^{-1}\right)\end{array}$ & $\begin{array}{c}\mathrm{DPR}^{\mathrm{d}} \\
(\%)\end{array}$ \\
\hline $\begin{array}{l}\text { Tecido } \\
\text { muscular } \\
\text { bovino }\end{array}$ & -a $^{\mathrm{a}}$ & NAA & $22,9 \pm 3,2(4)$ & 14,0 \\
$\begin{array}{l}\text { Tecido } \\
\text { muscular } \\
\text { bovino }\end{array}$ & - & ENAA & $17,9 \pm 1,6(8)$ & 8,9 \\
$\begin{array}{l}\text { Tecido } \\
\text { muscular } \\
\text { bovino }\end{array}$ & Extração em & ENAA & $16,9 \pm 0,2(3)$ & 1,2 \\
$\begin{array}{l}\text { Tecido } \\
\text { muscular } \\
\text { bovino }\end{array}$ & Extração com & ENAA & $16,2 \pm 0,5(3)$ & 3,1 \\
$\begin{array}{l}\text { Tecido } \\
\text { muscular } \\
\text { bovino }\end{array}$ & solventes & & & \\
$\begin{array}{l}\text { Fígado } \\
\text { bovino }\end{array}$ & - & EDXRF & $17,1 \pm 1,3(4)$ & 7,6 \\
$\begin{array}{l}\text { Fígado } \\
\text { bovino }\end{array}$ & - & & & \\
$\begin{array}{l}\text { Fígado } \\
\text { bovino }\end{array}$ & - & NAA & & \\
\hline
\end{tabular}

aindica que não foi realizada pré concentração/separação; 'bédia das concentrações e desvio padrão; ${ }^{c} n$ indica número de determinações; desvio padrão relativo.

Com relação à exatidão dos resultados obtidos por diferentes técnicas analíticas para a amostra de tecido muscular convém salientar a concordância existente entre os dados obtidos por ENAA, ENAA com pré-separação por meio de extração em fase sólida e por EDXRF. O valor médio da concentração de paládio considerando estas três técnicas é de $17,3 \pm 0,7 \mu \mathrm{g} \mathrm{g}^{-1}$. Na NAA foi obtido um valor bastante elevado quando comparado aos resultados obtidos pelas outras técnicas, devido à contribuição da interferência espectral de outros radioisótopos formados na irradiação. A con- centração obtida por ENAA após separação por extração com solventes foi ligeiramente mais baixa, devido a não recuperação total de paládio na pré-separação. Considerando o valor médio de $17,3 \pm 0,7 \mu \mathrm{g} \mathrm{g}^{-1}$ como sendo a concentração de Pd no tecido muscular, foram obtidos como rendimento de recuperação de $\mathrm{Pd}$ os valores de 93,6 e 97,7\% utilizando-se para separação a extração com solventes e a extração em fase sólida, respectivamente.

Nas análises da amostra de fígado bovino com Pd adicionado (Tabela 1) o Pd não foi detectado na NAA e determinou-se seu limite de detecção segundo o critério de Currie. . $^{31,32}$ Já os resultados obtidos para esta amostra por ENAA e EDXRF indicam uma boa concordância entre os valores obtidos por estas duas técnicas, bem como uma boa reprodutibilidade com desvios padrão relativos inferiores a $5,3 \%$.

Na Tabela 2 estão os resultados das médias das concentrações de Pd obtidos para o material catalisador automotivo do teste de proficiência do CCQM- P63, determinados pelos métodos instrumentais de NAA e ENAA. Estes resultados indicam uma boa reprodutibilidade, com desvios padrão relativos inferiores a $10,5 \%$. Como o valor certificado para Pd neste material ainda não foi divulgado, os resultados obtidos neste trabalho foram comparados com os valores preliminares apresentados por 21 dos laboratórios participantes no teste de proficiência, ${ }^{33}$ os quais também se encontram na Tabela 2. Os resultados obtidos pelos métodos instrumentais de NAA e ENAA estão dentro da faixa de valores apresentados pelos laboratórios participantes deste teste de proficiência. Aplicando a pré-separação química, os resultados obtidos foram ligeiramente inferiores que o valor médio preliminar dos dados apresentados pelos laboratórios participantes do teste de proficiência, devido a não recuperação total do Pd na etapa da separação.

Tabela 2. Médias das concentrações de Pd obtidas no material catalisador automotivo CCQM-P63, por diferentes métodos

\begin{tabular}{lcc}
\hline Método de análise & $\begin{array}{c}\text { Concentrações Pd } \\
\left(\mu \mathrm{g} \mathrm{g}^{-1}\right)\end{array}$ & $\begin{array}{c}\mathrm{DPR}^{\mathrm{b}} \\
(\%)\end{array}$ \\
\hline NAA & $2372 \pm 249(4)^{*}$ & 10,5 \\
ENAA & $2609 \pm 65(4)$ & 2,5 \\
ENAA após pré-separação & $1919 \pm 53(3)$ & 2,8 \\
por extração com solventes & & 9,1 \\
ENAA após pré-separação & $2200 \pm 200(3)$ & \\
por extração em fase sólida & & \\
\hline Resultados preliminares & & \\
& $\mathrm{X} \pm \mathrm{DP}^{33}\left(\mu \mathrm{g} \mathrm{g} \mathrm{g}^{-1}\right): 2708 \pm 96 ;$ \\
& Faixa $\left(\mu \mathrm{g} \mathrm{g}^{-1}\right): 2331-2780$ \\
\hline
\end{tabular}

*média das concentrações e desvio padrão. Os valores entre parênteses indicam número de determinações; ' média das concentrações e desvio padrão dos resultados obtidos por 21 laboratórios; 'bdesvio padrão relativo.

Para se ter uma idéia da sensibilidade das técnicas analíticas aplicadas neste trabalho para determinação de Pd foram avaliados os limites de detecção. Estes limites obtidos para análise de tecido muscular bovino, fígado bovino e catalisador automotivo estão apresentados na Tabela 3. Comparando os valores de limite de detecção obtidos por diferentes técnicas verifica-se, conforme esperado, a baixa sensibilidade obtida na NAA. Isto se deve a problemas de interferências espectrais decorrentes do efeito Compton do ${ }^{24} \mathrm{Na}$ e da radiação de freiamento do ${ }^{32} \mathrm{P}$. Já a ENAA proporciona um aumento da sensibilidade, devido ao alto valor da integral de ressonância do isótopo alvo ${ }^{108} \mathrm{Pd}$ de 253 barns e baixa seção de choque para nêutrons térmicos de 8,77 barns, e o sódio presente em alto teor na amostra apresenta baixo valor de integral de resso- 
Tabela 3. Limites de detecção de Pd obtidos nas análises de amostras de tecido muscular, fígado bovino e catalisador automotivo, por diferentes técnicas nucleares

\begin{tabular}{|c|c|c|c|}
\hline Amostra & $\begin{array}{c}\text { Técnica de } \\
\text { pré-separação }\end{array}$ & $\begin{array}{c}\text { Método } \\
\text { de } \\
\text { análise }\end{array}$ & $\begin{array}{c}\text { Limites } \\
\text { de detecção } \\
\left.(\mu \mathrm{g} \mathrm{kg})^{-1}\right)\end{array}$ \\
\hline $\begin{array}{l}\text { Tecido } \\
\text { muscular } \\
\text { bovino }\end{array}$ & $-{ }^{a}$ & NAA & 11000 \\
\hline $\begin{array}{l}\text { Tecido } \\
\text { muscular } \\
\text { bovino }\end{array}$ & - & ENAA & 413 \\
\hline $\begin{array}{l}\text { Tecido } \\
\text { muscular } \\
\text { bovino }\end{array}$ & $\begin{array}{l}\text { Extração em } \\
\text { fase sólida }\end{array}$ & ENAA & 68 \\
\hline $\begin{array}{l}\text { Tecido } \\
\text { muscular } \\
\text { bovino }\end{array}$ & $\begin{array}{l}\text { Extração com } \\
\text { solventes }\end{array}$ & ENAA & 93 \\
\hline $\begin{array}{l}\text { Tecido } \\
\text { muscular } \\
\text { bovino }\end{array}$ & - & EDXRF & 1000 \\
\hline $\begin{array}{l}\text { Fígado } \\
\text { bovino }\end{array}$ & - & NAA & 12000 \\
\hline $\begin{array}{l}\text { Fígado } \\
\text { bovino }\end{array}$ & - & ENAA & 370 \\
\hline $\begin{array}{l}\text { Fígado } \\
\text { bovino }\end{array}$ & - & EDXRF & 1000 \\
\hline $\begin{array}{l}\text { Catalisador } \\
\text { automotivo }\end{array}$ & - & ENAA & 13700 \\
\hline $\begin{array}{l}\text { Catalisador } \\
\text { automotivo }\end{array}$ & $\begin{array}{l}\text { Extração em } \\
\text { fase sólida }\end{array}$ & ENAA & 5400 \\
\hline
\end{tabular}

aindica que não foi realizada pré-separação.

nância. Fazendo a pré-separação do Pd para posterior ENAA, foi verificado o aumento da sensibilidade em relação aos métodos instrumentais de análise, sendo que a separação por extração em fase sólida foi a que proporcionou menor valor para limite de detecção. Os valores de limite de detecção obtido para o material catalisador automotivo foram superiores aos daqueles obtidos para amostras biológicas. Os limites de detecção dependem do tipo de matriz e, no caso, o material catalisador automotivo é uma matriz de composição bastante complexa.

\section{CONCLUSÕES}

Os resultados obtidos neste trabalho permitiram concluir que a ENAA é mais apropriada para a determinação de Pd em materiais biológicos. Na ENAA com a redução do problema de interferências espectrais principalmente da alta atividade do ${ }^{24} \mathrm{Na}$ e do ${ }^{32} \mathrm{P}$, os dados obtidos indicaram uma boa precisão e alta sensibilidade quando comparados com aqueles encontrados na NAA e EDXRF.

A introdução da etapa da separação do Pd antes da ENAA proporcionou a obtenção de resultados precisos e baixos valores de limites de detecção em comparação com a ENAA instrumental.

No que diz respeito à exatidão dos resultados obtidos, torna-se difícil fazer uma comparação entre os métodos utilizados visto não se dispor de materiais biológicos de referência com valores certificados para Pd. Desta maneira, para avaliar a exatidão foi feito um estudo comparativo entre os resultados obtidos por diferentes técnicas aplicadas neste trabalho e no caso do material catalisador do teste de proficiência foi possível comparar também com o valor preliminar apresentado pelos laboratórios participantes da intercomparação.

Nas análises dos materiais biológicos, pôde se concluir que houve, de modo geral, uma boa concordância entre as médias das determinações de Pd obtidas por diferentes métodos. Também no caso dos resultados de Pd obtidos no material catalisador automotivo do teste de proficiência houve uma boa concordância dos dados obtidos por NAA e ENAA, e com o valor médio preliminar da intercomparação. Isto é, os resultados obtidos pelos métodos instrumentais (NAA e ENAA) aproximaram-se mais deste valor médio preliminar.

Os resultados obtidos por meio da ENAA após a aplicação das técnicas de separação de Pd tanto para amostras biológicas como no material catalisador automotivo foram ligeiramente mais baixos que aqueles obtidos pelos métodos puramente instrumentais, indicando a necessidade de determinar o rendimento da separação química para fazer as devidas correções. Na aplicação dos métodos de separação química, devido ao manuseio da amostra durante o processamento, podem ocorrer perdas de Pd por transferências entre frascos e por adsorção nas paredes de recipientes. Na separação química, ao mesmo tempo em que se eliminam os elementos interferentes, pode ocorrer perda do elemento de interesse.

\section{AGRADECIMENTOS}

Ao CNPq e à FAPESP pelo suporte financeiro e ao Dr. J. R. Martinelli do Centro de Ciências e Tecnologia de Materiais (CCTM), IPEN-CNEN/SP, por ter concedido a utilização do equipamento para análise por fluorescência de raios $\mathrm{X}$.

\section{REFERÊNCIAS}

1. Wong, E.; Giandomenico, C. M.; Chem. Rev. 1999, 99, 2451.

2. Caires, A. F.; Almeida, E. T.; Mauro, A. E.; Hemerly, J. P.; Valentini, S. R.; Quim. Nova 1999, 22, 329.

3. Schwarzer, M.; Schuster, M.; Von Hentig, R.; Fresenius J. Anal. Chem. 2000, 368, 240.

4. Caramella-Crespi, V.; Pisami, U.; Ganzerli-Valentini, M. T.; Meloni, S.; Maxia, V.; J. Radioanal. Nucl. Chem. 1974, 23, 23.

5. Posta, S.; Kukula, F.; Radioisotopy 1976, 17, 559.

6. Byrne, A R.; Mikrochim. Acta 1981, 323.

7. Shah, N. K.; Wai, C. M.; J. Radioanal. Nucl. Chem., Lett. 1985, 94, 129.

8. Dybczynski, R.; Majchrzak, J.; Stokowska, H.; Szyszko, H.; Chem. Anal.(Warsaw, Pol.) 1990, 35, 609.

9. Begerow, J.; Turfeld, M.; Dunemann, L.; J. Anal. At. Spectrom. 1997, 12, 1095 .

10. Begerow, J.; Turfeld, M.; Dunemann, L.; Fresenius J. Anal. Chem. 1997, 359, 427.

11. Schramel, P.; Wendler, I.; Angerer, J.; Int. Arch. Occup. Environ. Health 1997, 69, 219.

12. Pyrzynska, K.; Talanta 1998, 47, 841.

13. Lesniewska, B. A.; Godlewska-Zylkiewicz, B.; Hulanicki, A.; Chem. Anal. (Warsaw, Pol.) 2005, 50, 945.

14. Morcelli, C. P. R.; Figueiredo, A. M. G.; Enzweiler, J.; Sarkis, J. E. S.; Jorge, A. P. S.; Kakazu, M.; Geostand Geoanal. Res. 2004, 28.

15. Morcelli, C. P. R.; Figueiredo, A. M. G.; Sarkis, J. E. S.; Enzweiler, J.; Kakazu, M.; Sigolo, J. B.; Sci. Total Environ. 2005, 345, 81,

16. Polák, J.; Mestek, O.; Suchánek, M.; J. for Quality, Comparability and Reliability in Chemical Measurement 2006, 10, 627.

17. Gao, J.; Peng, B.; Fan, H.; Kang, J.; Wang, X. S.; Talanta 1997, 44, 837.

18. Goldlewska-Zylkiewicz, B.; Lesniewska, B. A.; Gasiewska, U.; Hulanicki, A.; Anal. Lett. 2000, 33, 2805,

19. Boch, K.; Schuster, M.; Risse, G.; Schwarzer, M.; Anal. Chim. Acta 2002, 459, 257.

20. Zheng, H.; Li, H.; Zeng, Y.; Ma, L.; Yankuang Ceshi 2005, 24, 299.

21. Geldmacher-von Mallinckrodt, M.; Pooth, M.; Arch. Toxicol. 1969, 25, 5.

22. Goldlewska-Zylkiewicz, B.; Zaleska, M.; Anal. Chim. Acta 2002, 462, 305

23. Iglesias, M.; Anticó, E.; Salvadó, V.; Talanta 2003, 59, 651.

24. Chwastowska, J.; Skwara, W.; Sterlinska, E.; Pszonicki, L.; Talanta 2004, 64, 224. 
25. Gertler, A. W.; Isr. J. Chem. 1994, 34, 425.

26. Lu, Z.; Chow, J.; Watson, J.; Frazier, C.; Pritchett, L.; Dippel, W.; Bates, B.; Jones, W.; Torres, G.; Fisher, R.; Lam, D.; Proc. Annu. Meet Air Waste Manage. Assoc. 1994, 7, 1.

27. Kumar, S.; Verma, R.; Gangadharan, S.; Analyst 1993, 118, 1085.

28. Hodge, V. F.; Stallard, M. O.; Environ. Sci. Technol. 1986, 20, 1058.

29. IAEA; Nuclear analytical methods for platinum group elements, IAEATECDOC-1443, International Atomic Energy Agency: Vienna, 2005, p. 24.
30. Angelone, M.; Pinto, V.; Nardi, E.; Cremisini, C. Em Palladium emissions in the environment analytical methods, environmental assessment and health effects; Zereini, F.; Alt, F., eds.; Springer Verlag: Berlin, 2006, p. 455-485.

31. Currie, L. A.; Anal. Chem. 1968, 40, 586.

32. Currie, L. A.; Anal. Chim. Acta 1999, 391, 105.

33. CCQM; P63 Platinum group elements in an automobile catalyst, Teste de Proficiência, Wolf-Briche, C. coord.; LGC Reino Unido, Resultados preliminares, comunicação pessoal. 